\title{
Local density of electromagnetic states within a nanometric gap formed between two thin films supporting surface phonon polaritons
}

\author{
Mathieu Francoeur, ${ }^{1, a)}$ M. Pinar Mengüçc, ${ }^{1,2, a)}$ and Rodolphe Vaillon ${ }^{3, b)}$ \\ ${ }^{1}$ Department of Mechanical Engineering, Radiative Transfer Laboratory, University of Kentucky, \\ Lexington, Kentucky 40506-0503, USA \\ ${ }^{2}$ Ozyegin University, Altunizade, Uskudar, 34662 Istanbul, Turkey \\ ${ }^{3}$ CNRS, INSA-Lyon, UCBL, CETHIL, Université de Lyon, UMR5008, F-69621 Villeurbanne, France
}

(Received 24 June 2009; accepted 15 December 2009; published online 9 February 2010)

\begin{abstract}
We present a detailed physical analysis of the near-field thermal radiation spectrum emitted by a silicon carbide $(\mathrm{SiC})$ film when another nonemitting $\mathrm{SiC}$ layer is brought in close proximity. This is accomplished via the calculation of the local density of electromagnetic states (LDOS) within the gap formed between the two thin films. An analytical expression for the LDOS is derived, showing explicitly that (i) surface phonon polariton (SPhP) coupling between the layers leads to four resonant modes, and (ii) near-field thermal radiation emission is enhanced due to the presence of the nonemitting film. We study the impact of the interfilm separation gap, the distance where the fields are calculated, and the thickness of the nonemitting layer on the spectral distribution of the LDOS. Results show that for an interfilm gap of $10 \mathrm{~nm}$, the near-field spectrum emitted around the SPhP resonance can increase more than an order of magnitude as compared to a single emitting thin layer. Interfilm SPhP coupling also induces a loss of spectral coherence of resonance, mostly affecting the low frequency modes. The effect of the nonemitting film can be observed on LDOS profiles when the distance where the fields are calculated is close to the interfilm gap. As the LDOS is calculated closer to the emitter, the near-field spectrum is dominated by SPhPs with small penetration depths that do not couple with the modes associated with the nonemitting film, such that thermal emission is similar to what is observed for a single emitting layer. Spectral distribution of LDOS is also significantly modified by varying the thickness of the nonemitting film relative to the thickness of the emitting layer, due to an increasing mismatch between the cross-coupled SPhP modes. The results presented here show clearly that the resonant modes of thermal emission by a polar crystal can be enhanced and tuned, between the transverse and longitudinal optical phonon frequencies, by simply varying the structure of the system. This analysis provides the physical grounds to tune near-field thermal radiation emission via multilayered structures, which can find application in nanoscale-gap thermophotovoltaic power generation. () 2010 American Institute of Physics.
\end{abstract}

[doi:10.1063/1.3294606]

\section{INTRODUCTION}

Near-field radiative heat transfer by and between bulk materials was extensively studied over the past years. On the other hand, attention was paid only recently to near-field thermal radiation emission by nanometric films supporting surface waves. ${ }^{1-8}$ When the emitter of thermal radiation is thin, surface waves associated with each interface couple within the layer and split the single resonance into antisymmetric and symmetric modes, a phenomenon that was investigated in the past for both surface phonon polaritons (SPhPs) and surface plasmon polaritons (SPPs). ${ }^{9-16}$ This resonance splitting affects the spectrum emitted and can thus be used to tune near-field thermal radiation emission; such control over the near-field spectrum emitted can find application in nanoscale-gap thermophotovoltaic (TPV) power generation. $^{17,18}$

\footnotetext{
${ }^{a)}$ Tel.: (859) 257-6336 ext. 80696. FAX: (859) 257-3304. Electronic addresses: mfran0@engr.uky.edu, menguc@engr.uky.edu and pinar.menguc@ozyegin.edu.tr.

b) Tel.: +33 (0)4 724388 17. FAX: +33 (0)4 724388 11. Electronic mail: rodolphe.vaillon@insa-lyon.fr.
}

Biehs et al. ${ }^{1,2}$ studied emission from thin metallic films and materials coated with metal films, and reported that the single SPP resonance at a bulk metal-vacuum interface is split into two frequencies due to the coupling of SPPs inside the layer, thus affecting the local density of electromagnetic states (LDOS) above the film in vacuum. Similar observations were made by Joulain and Henkel, ${ }^{3}$ who discussed the spatial correlation spectrum of near-field thermal radiation above a thin metallic film. Drevillon ${ }^{4}$ provided a preliminary study of the near-field spectrum emitted by single and multiple thin films made of polar crystals and metals, without giving detailed physical explanations. This work was extended by Ben-Abdallah et al., ${ }^{5}$ who analyzed the possibility of tailoring near-field LDOS spectra above one-dimensional metallodielectric media made of aluminum (Al) and lossless dielectric layers. A target LDOS was prescribed and a genetic algorithm was used to find the best structure, operating in the near ultraviolet, leading to that LDOS profile. While this work showed that it is possible to tune the near-field thermal radiation spectra via multilayered structures, little information is provided about the physical details and on how the interfilm coupling of surface waves affects the LDOS pro- 
files calculated. Fu and $\operatorname{Tan}^{6}$ studied near-field radiative heat transfer between two bulks with one of the material coated with a film supporting SPhPs. They provided extensive parametric analyses of the influence of the coating (material and thickness) on the total radiative heat flux values for TE- and TM-polarized modes both in the near- and far-field regimes; on the other hand, the alteration of the spectral distribution of radiative flux was not investigated. Francoeur et al. ${ }^{7}$ studied near-field thermal radiation between a silicon carbide $(\mathrm{SiC})$ nanometric film and a bulk, and found a narrow spectral band enhancement of the radiative heat flux due to $\mathrm{SPhP}$ coupling inside the layer. Recently, Ben-Abdallah et al. ${ }^{8}$ investigated near-field radiative heat transfer between two films supporting SPhPs. The authors found that for films of equal thicknesses, the total radiative heat transfer coefficient $h_{r}$ varies as $d^{-2}$ (where $d$ is the separation gap between the films) as for the case of two bulks, while for layers of different thicknesses, $h_{r}$ varies as $d^{-3}$. However, the physical details of this behavior and the spectral variations of $h_{r}$ as a function of the structure of the system were not systematically addressed.

From the above discussion, it is clear that there is a need to study the physics of near-field emission involving thin layers supporting surface waves. The objective of this paper is therefore to analyze thoroughly the physics of near-field thermal emission by a thin film supporting surface polaritons in the presence of a nonemitting body, also supporting surface waves, in close proximity. This task is accomplished by calculating the LDOS within the gap formed by two SiC films supporting SPhPs in the infrared region. The manuscript is structured as follows: an analytical expression for the LDOS within the gap separating the thin layers is first derived; for this purpose, the Maxwell equations combined with fluctuational electrodynamics are used. Then, the crosscoupled resonant modes for the two film system are investigated via calculation of the dispersion relation. The impact of interfilm coupling on emitted near-field thermal radiation spectra is afterward outlined via computation of the LDOS for various interfilm separation gaps, distances where the fields are observed, and layer thicknesses. Finally, concluding remarks are provided.

\section{MATHEMATICAL FORMULATION OF THE LDOS IN THE GAP FORMED BETWEEN TWO THIN FILMS}

The geometry considered is schematically depicted in Fig. 1, where a polar $(\rho, \theta, z)$ coordinate system is shown.

The media are infinite along the $\rho$-direction and invariant over $\theta$ (i.e., azimuthal symmetry), such that only variations along the $z$-axis need to be considered. Throughout this paper, it is assumed that the films are in local thermodynamic equilibrium, homogeneous, isotropic, nonmagnetic, and described by a frequency-dependent dielectric function $\varepsilon_{r}(\omega)$, which is local in space. Also, the surfaces of the layers are considered parallel and perfectly smooth. The two polar crystal films, labeled media 1 and 3 with thicknesses $t_{1}$ and $t_{3}$, respectively, are submerged in vacuum and separated by a gap of length $d_{c}$. Layer 1 , at prescribed temperature $T_{1}$, emits thermal radiation while medium 3 is assumed to be nonemit-

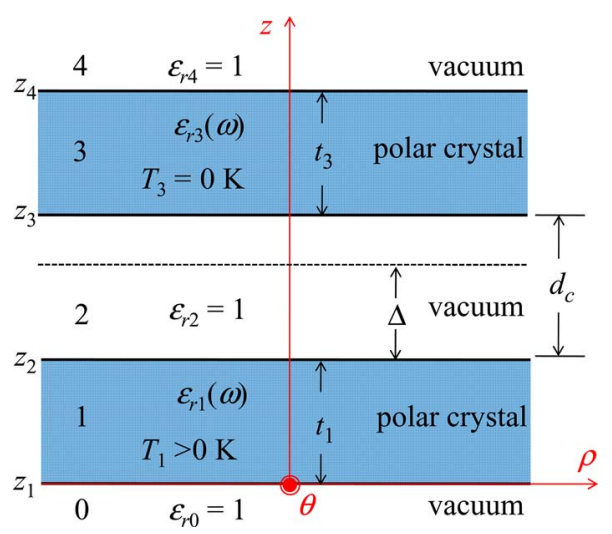

FIG. 1. (Color online) Schematic representation of the geometry considered: The LDOS is calculated at a distance $\Delta$ above the emitting film 1 within the gap of thickness $d_{c}$.

ting. The thermal radiation field is calculated in the gap at a distance $\Delta$ above interface 1-2. The assumption of a nonemitting film is justified by the fact that when calculating radiation transfer between two layers, the energy emitted by one of the media absorbed by the other layer is calculated, and vice versa. The difference between these two values gives the net radiant energy exchanged; therefore, understanding the physics of thermal radiation emission from one film with $T>0 \mathrm{~K}$, while the other one is nonemitting, allows the interpretation of the realistic case of near-field radiative heat transfer between two emitting films.

The emitted near-field thermal radiation spectrum is analyzed via the calculation of the LDOS, which is determined by normalizing the electromagnetic energy density at location $\Delta$ by the mean energy of a state $\Theta\left(\omega, T_{1}\right)$. This is justified by the fact that we are investigating the LDOS in the frequency range between $1.5 \times 10^{14}$ and $1.9 \times 10^{14} \mathrm{rad} / \mathrm{s}$, corresponding to frequencies excited in typical thermal radiation applications involving temperatures between 300 and $2000 \mathrm{~K}$.

The LDOS in the near field is calculated starting from the Maxwell equations combined with fluctuational electrodynamics, where the source of thermal radiation is modeled as a stochastic current density. ${ }^{19,20}$ The monochromatic LDOS at location $\Delta$ above film 1 in medium 2 can be written as follows after application of the fluctuation-dissipation theorem:

$$
\begin{aligned}
\rho_{\omega, 12}(\Delta)= & \frac{\omega \varepsilon_{r 1}^{\prime \prime}(\omega)}{2 \pi^{2} c_{v}^{2}} \int_{0}^{\infty} k_{\rho} d k_{\rho} \int_{z_{1}}^{z_{2}} d z^{\prime} \\
& \times\left(k_{v}^{2}\left|g_{12 m \alpha}^{E}\left(k_{\rho}, \Delta, z^{\prime}, \omega\right)\right|^{2}\right. \\
& \left.+\left|g_{12 m \alpha}^{H}\left(k_{\rho}, \Delta, z^{\prime}, \omega\right)\right|^{2}\right),
\end{aligned}
$$

where the subscripts $m$ and $\alpha$ involve summation over the components $\rho, \theta$, and $z$. The terms $g_{12 m \alpha}^{E}$ and $g_{12 m \alpha}^{H}$ are the plane wave representation (Weyl components) of the electric and magnetic dyadic Green's functions (DGFs), relating the fields observed at $\Delta$ in medium 2 with frequency $\omega$ and wavevector $k_{\rho}$ to a source $z^{\prime}$ located in layer 1 . The electric Weyl representation of the DGF is given by ${ }^{21}$ 


$$
\begin{aligned}
\overline{\mathbf{g}}_{12}^{E}\left(k_{\rho}, \Delta, z^{\prime}, \omega\right)= & \frac{i}{2 k_{z 1}}\left[\left(A_{2}^{\mathrm{TE}} \hat{\mathbf{s}} \hat{\mathbf{s}}+A_{2}^{\mathrm{TM}} \hat{\mathbf{p}}_{2}^{+} \hat{\mathbf{p}}_{1}^{+}\right) e^{i\left[k_{z 2} \Delta-k_{z 1} z^{\prime}\right]}\right. \\
& +\left(B_{2}^{\mathrm{TE}} \hat{\mathbf{s}} \hat{\mathbf{s}}+B_{2}^{\mathrm{TM}} \hat{\mathbf{p}}_{2}^{-} \hat{\mathbf{p}}_{1}^{+}\right) e^{i\left[-k_{z 2} \Delta-k_{z 1} z^{\prime}\right]} \\
& +\left(C_{2}^{\mathrm{TE}} \hat{\mathbf{s}} \hat{\mathbf{s}}+C_{2}^{\mathrm{TM}} \hat{\mathbf{p}}_{2}^{+} \hat{\mathbf{p}}_{1}^{-}\right) e^{i\left[k_{z 2} \Delta+k_{z 1} z^{\prime}\right]} \\
& \left.+\left(D_{2}^{\mathrm{TE}} \hat{\mathbf{s}} \hat{\mathbf{s}}+D_{2}^{\mathrm{TM}} \hat{\mathbf{p}}_{2}^{-} \hat{\mathbf{p}}_{1}^{-}\right) e^{i\left[-k_{z 2} \Delta+k_{z 1} z^{\prime}\right]}\right]
\end{aligned}
$$

where TE- and TM-polarized unit vectors, defined as $\hat{\mathbf{s}}=-\hat{\boldsymbol{\theta}}$ and $\hat{\mathbf{p}}_{i}^{ \pm}=\left(k_{\rho} \hat{\mathbf{z}} \mp k_{z i} \hat{\boldsymbol{\rho}}\right) / k_{i}$, have been used. ${ }^{22}$ The magnetic counterpart of Eq. (2) is calculated as $\overline{\mathbf{g}}_{12}^{H}=\nabla \times \overline{\mathbf{g}}_{12}^{E}$. The coefficients $A_{2}^{\gamma}$ and $B_{2}^{\gamma}$ represent amplitudes of forward ( $z$-positive) and backward (z-negative) traveling waves, respectively, in layer 2 and polarization state $\gamma$, arising from a source emitting in the forward direction. Similar explanations hold for $C_{2}^{\gamma}$ and $D_{2}^{\gamma}$, except that these coefficients arise from a source emitting in the backward direction. ${ }^{21}$ Using a transfer matrix approach, ${ }^{23}$ the coefficients in medium 2 are given by

$$
\begin{aligned}
& A_{2}^{\gamma}=\frac{e^{i k_{z 1} t_{1}} t_{12}^{\gamma}}{\left(1+r_{01}^{\gamma} r_{12}^{\gamma} e^{2 i k_{z 1} t_{1}}\right)\left(1-R_{1}^{\gamma} R_{3}^{\gamma} e^{\left.2 i k_{z 2} d_{c}\right)}\right.}, \\
& B_{2}^{\gamma}=R_{3}^{\gamma} e^{2 i k_{z 2} d_{c} A_{2}^{\gamma},} \\
& C_{2}^{\gamma}=r_{34}^{\gamma} A_{2}^{\gamma}, \\
& D_{2}^{\gamma}=r_{34}^{\gamma} R_{3}^{\gamma} e^{2 i k_{z 2} d_{c}} A_{2}^{\gamma},
\end{aligned}
$$

where $r_{j, j+1}^{\gamma}$ and $t_{j, j+1}^{\gamma}$ are, respectively, the Fresnel reflection and transmission coefficients at the interface delimiting media $j$ and $j+1$ in polarization state $\gamma$, while $R_{j}^{\gamma}$ is the reflection coefficient of layer $j$ in polarization state $\gamma$ given by ${ }^{23}$

$$
R_{j}^{\gamma}=\frac{r_{j-1, j}^{\gamma}+r_{j, j+1}^{\gamma} e^{2 i k_{z j} t_{j}}}{1+r_{j-1, j}^{\gamma} r_{j, j+1}^{\gamma} e^{2 i k_{z j} t_{j}}} .
$$

The coefficients given by Eqs. (3a)-(3d) are then included in the electric and magnetic Weyl components of the DGF, which are in turn substituted in Eq. (1). Since SPhPs exist only in TM polarization for nonmagnetic media, ${ }^{16}$ we consider strictly the TM evanescent component of the LDOS. After plenty of algebraic manipulations, the following compact expression for the TM evanescent component of the monochromatic LDOS is obtained:

$$
\begin{aligned}
\rho_{\omega, 12}^{\operatorname{evan}, \mathrm{TM}}(\Delta)= & \frac{1}{2 \pi^{2} \omega} \int_{k_{v}}^{\infty} \frac{k_{\rho}^{3} d k_{\rho}}{\left|k_{z 2}\right|} \frac{\operatorname{Im}\left(R_{1}^{\mathrm{TM}}\right) e^{-2 k_{z 2}^{\prime \prime} \Delta}}{\mid 1-R_{1}^{\mathrm{TM}} R_{3}^{\mathrm{TM}} e^{\left.2 i k_{z 2} d_{c}\right|^{2}}} \\
& \times\left[\mid 1+R_{3}^{\mathrm{TM}} e^{-\left.2 k_{z 2}^{\prime \prime}\left(d_{c}-\Delta\right)\right|^{2}}\right. \\
& \left.-2 \frac{\left|k_{z 2}\right|^{2}}{k_{\rho}^{2}} \operatorname{Re}\left(R_{3}^{\mathrm{TM}}\right) e^{-2 k_{z 2}^{\prime \prime}\left(d_{c}-\Delta\right)}\right] .
\end{aligned}
$$

Such an analytical expression for the LDOS within the gap between two films has never been reported in literature, and it is therefore important to analyze its physical meaning. The denominator $\left|1-R_{1}^{\mathrm{TM}} R_{3}^{\mathrm{TM}} e^{2 i k_{z 2} d_{c}}\right|^{2}$ accounts for multiple reflection and wave interference between the two films, while the term in the numerator, $\operatorname{Im}\left(R_{1}^{\mathrm{TM}}\right)$, can be seen as the spec- tral near-field emittance of film 1 . The evanescent nature of these modes is also explicitly shown via the exponentially decaying term $e^{-2 k_{z 2}^{\prime \prime} \Delta}$. The last term in square bracket on the right-hand side of Eq. (5) accounts for the increase in the emittance of film 1 due to the presence of layer 3. It can be seen via the exponential term $e^{-2 k_{z 2}^{\prime \prime}\left(d_{c}-\Delta\right)}$ that the influence of film 3 on the near-field thermal radiation spectrum emitted is maximal when $\Delta=d_{c}$ and minimal when $\Delta \rightarrow 0$.

In the limiting case that $d_{c} \rightarrow \infty, e^{-2 k_{z 2}^{\prime \prime} d_{c}} \rightarrow 0$ such that both the denominator and the term in square bracket in Eq. (5) tend to 1. The TM evanescent component of the monochromatic LDOS above a single emitting film is then retrieved as follows:

$$
\rho_{\omega, 12}^{\operatorname{evan}, \mathrm{TM}}(\Delta)=\frac{1}{2 \pi^{2} \omega} \int_{k_{v}}^{\infty} \frac{k_{\rho}^{3} d k_{\rho}}{\left|k_{z 2}\right|} \operatorname{Im}\left(R_{1}^{\mathrm{TM}}\right) e^{-2 k_{z 2}^{\prime \prime} \Delta} .
$$

Moreover, if medium 1 is thick (i.e., bulk), then $t_{1} \rightarrow \infty$, $e^{i k_{z 1} t_{1}} \rightarrow 0$, and $R_{1}^{\mathrm{TM}} \rightarrow r_{21}^{\mathrm{TM}}$. Substitution of $\operatorname{Im}\left(r_{21}^{\mathrm{TM}}\right)$ in Eq. (6), instead of $\operatorname{Im}\left(R_{1}^{\mathrm{TM}}\right)$, provides the LDOS at distance $\Delta$ above an emitting bulk as reported by Joulain. ${ }^{24}$

\section{RESULTS}

\section{A. Dispersion relation of cross-coupled SPhPs}

Since medium 1 is at a finite temperature $\left(T_{1} \neq 0 \mathrm{~K}\right)$, thermal excitation of transverse optical phonons generate SPhPs at each polar crystal-vacuum interfaces 0-1 and 1-2 with evanescent fields decaying in both media. SPhPs are also excited at interfaces 2-3 and 3-4 of medium 3, but via different mechanisms. SPhPs at interface 2-3 are excited via tunneling of evanescent waves generated by the emitting layer, which is analog to the Otto configuration. ${ }^{16}$ At interface 3-4, excitation of SPhPs is done via tunneling of evanescent waves of interface 2-3 through film 3, similar to the Kretschmann configuration. ${ }^{16}$ When both $T_{1}$ and $T_{3}$ are greater than $0 \mathrm{~K}, \mathrm{SPhP}$ excitation for each layer is due to thermal excitation of charges, as well as excitation via Otto and Kretschmann configurations.

$\mathrm{SPhP}$ dispersion relation can be determined by analyzing the resonance condition of the two film system. Resonance arises when $\rho_{\omega, 12}^{\text {evan,TM }} \rightarrow \infty$. Inspection of Eq. (5) shows that divergence of the TM evanescent component of the monochromatic LDOS can only happen when the following condition is fulfilled:

$$
1-R_{1}^{\mathrm{TM}} R_{3}^{\mathrm{TM}} \exp \left(2 i k_{z 2} d_{c}\right)=0 .
$$

If medium 3 is removed, the TM evanescent component of the LDOS is given by Eq. (6), and $\rho_{\omega, 12}^{\text {evan,TM }} \rightarrow \infty$ is satisfied when $1-\left(r_{21}^{\mathrm{TM}}\right)^{2} \exp \left(2 i k_{z 1} t_{1}\right)=0$. Solution of this last expression provides the resonant modes of a single film submerged in a given medium. Moreover, if the emitting layer 1 is thick (i.e., bulk), the single resonance condition is given by $r_{21}^{\mathrm{TM}}$ $\rightarrow \infty$. Using the definition of the Fresnel reflection coefficient in TM polarization, this condition is fulfilled when $\varepsilon_{r 1} k_{z 2}$ $+\varepsilon_{r 2} k_{z 1}=0$, which corresponds to the resonant mode of a single interface delimiting media 1 and $2 .^{25}$

$\mathrm{SPhP}$ dispersion relation for the two film system leads to four branches, and is determined by solving Eq. (7) using the 

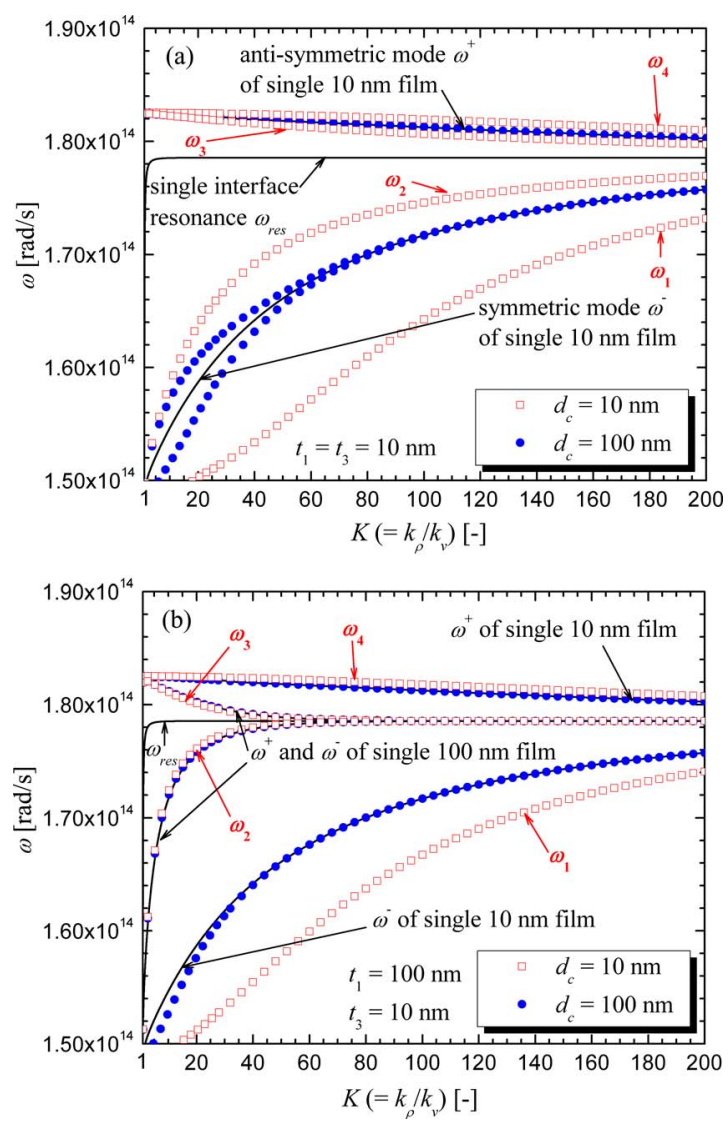

FIG. 2. (Color online) Dispersion relations of SPhPs for $t_{1}$ and $t_{3}$ thick SiC films submerged in vacuum and separated by a gap $d_{c}$ : (a) $t_{1}=t_{3}=10 \mathrm{~nm}$, and $d_{c}=10$ and $100 \mathrm{~nm}$. (b) $t_{1}=100 \mathrm{~nm}, t_{3}=10 \mathrm{~nm}$, and $d_{c}=10$ and $100 \mathrm{~nm}$. The results are compared with the dispersion relations of single $\mathrm{SiC}$ films in vacuum and a single $\mathrm{SiC}$-vacuum interface.

secant method. For the simulations, the dielectric constant of polar crystals is approximated by a damped harmonic oscillator model given by $\varepsilon_{r}(\omega)=\varepsilon_{\infty}\left(\omega^{2}-\omega_{\mathrm{LO}}^{2}+i \Gamma \omega\right) /\left(\omega^{2}-\omega_{\mathrm{TO}}^{2}\right.$ $+i \Gamma \omega)$, with parameters $\varepsilon_{\infty}=6.7, \omega_{\mathrm{LO}}=1.825 \times 10^{14} \mathrm{rad} / \mathrm{s}$, $\omega_{\mathrm{TO}}=1.494 \times 10^{14} \mathrm{rad} / \mathrm{s}$, and $\Gamma=8.966 \times 10^{11} 1 / \mathrm{s}$ for SiC. ${ }^{26}$ Note that the losses are neglected when calculating the dispersion relation (i.e., $\Gamma=0$ ); the full damped harmonic oscillator is, however, used when computing the LDOS.

In Fig. 2(a), dispersion relations for two $10 \mathrm{~nm}$ thick SiC films spaced by a variable $d_{c}$ of 10 and $100 \mathrm{~nm}$ are shown, while $\mathrm{SPhP}$ dispersion relations for $t_{1}=100 \mathrm{~nm}, t_{3}=10 \mathrm{~nm}$, and $d_{c}=10$ and $100 \mathrm{~nm}$ are presented in Fig. 2(b). The four branches are identified as a function of their frequencies; the lowest frequency branch is referred to as $\omega_{1}$, while the highest one is called $\omega_{4}$. For clarity, this nomenclature is shown in Figs. 2(a) and 2(b) only for $d_{c}=10 \mathrm{~nm}$. In both figures, results are compared with the dispersion relations of single $\mathrm{SiC}$ films in vacuum and a single SiC-vacuum interface.

In a thin film, the evanescent field of SPhPs associated with each interface can interact with each other, thus leading to a splitting of the $\mathrm{SPhP}$ dispersion relation into antisymmetric $\omega^{+}$(high frequency) and symmetric $\omega^{-}$(low frequency) modes. ${ }^{9-16}$ The symmetric mode corresponds to the case where the tangential electric field $E_{\rho}$ has a symmetric distribution with respect to the middle plane of the layer (magnetic fields $H_{\theta}$ at each interface are in phase), and vice versa for the antisymmetric resonance. ${ }^{15}$ Comparison of the dispersion relations for 10 and $100 \mathrm{~nm}$ thick single SiC films in vacuum shows that the splitting of the resonance becomes more pronounced as the thickness of the film decreases. For large $K\left(=k_{\rho} / k_{v}\right)$ values, the dispersion relations of both modes approach asymptotically the dispersion curve of a single SiC-vacuum interface. Indeed, the penetration depth of SPhPs in the film is small for large $K$ values. As a consequence, when $K$ is large, the antisymmetric and symmetric branches become degenerate as SPhPs do not couple inside the films, and therefore, the SPhPs at each interface behave independently of each other. ${ }^{12}$

When two films supporting SPhPs are placed in close proximity, further coupling takes place, and the dispersion relation splits into four branches showing antisymmetric and symmetric resonances for each film and for the entire structure. $^{27,28}$ This fact is illustrated by analyzing the case $d_{c}=10 \mathrm{~nm}$ in Fig. 2(a), where both films are $10 \mathrm{~nm}$ thick. The modes $\omega_{1}$ and $\omega_{2}$ come from the symmetric resonance of each film. The interaction of these symmetric modes in the vacuum gap leads to antisymmetric $\left(\omega_{2}\right)$ and symmetric resonances $\left(\omega_{1}\right)$ for the whole structure. The antisymmetric resonance $\omega_{2}$ arises when $H_{\theta}$ oscillations, in phase at the two interfaces of a given layer, are out of phase from one film relative to the other one. Similarly, the symmetric resonance $\omega_{1}$ due to interfilm coupling arises when $H_{\theta}$ at the four interfaces are in phase. It can be seen in Fig. 2(a) that as $d_{c}$ increases, $\omega_{1}$ and $\omega_{2}$ get closer to the symmetric branch of a single $10 \mathrm{~nm}$ thick film submerged in vacuum, since when $d_{c}$ is large enough, SPhPs on each film behave independently. Similar explanations are applicable to $\omega_{3}$ and $\omega_{4}$, which are generated by the coupling of the antisymmetric modes of each film. For the $d_{c}$ values considered in Fig. 2(a), $\omega_{3}$ and $\omega_{4}$ are very close to each other; the proximity effect between the films is more visible for the modes $\omega_{1}$ and $\omega_{2}$. For films of different thicknesses [Fig. 2(b)], $\omega_{4}$ is located above $\omega^{+}$of the thinner film $(10 \mathrm{~nm})$, and $\omega_{3}$ is below $\omega^{+}$of the thicker film $(100 \mathrm{~nm})$. Similar observations can be made for $\omega_{1}$ and $\omega_{2}$, which are, respectively, located below and above $\omega^{-}$of 10 and $100 \mathrm{~nm}$ thick films. Note that due to the symmetry of the problem, SPhP dispersion relation for $t_{1}=10 \mathrm{~nm}$ and $t_{3}$ $=100 \mathrm{~nm}$ is the same as the one presented in Fig. 2(b). For sufficiently large $K$ values where SPhP coupling is impossible in each layer and between the films, all four branches converge toward the resonant frequency $\omega_{\text {res }}$ of a single SiCvacuum interface.

\section{B. LDOS profiles within the gap formed between the two films}

The near-field thermal radiation spectrum emitted by film 1 is analyzed by calculating the TM evanescent component of the monochromatic LDOS given by Eq. (5). These LDOS profiles are calculated in the vacuum gap at distance $\Delta$ above layer 1 , such that when $\Delta=d_{c}$, this implies that the LDOS is computed just before crossing interface 2-3 (i.e., at $z=z_{3}^{-}$). Following the discussion of Sec. III A, the near-field thermal radiation spectrum emitted is expected to be strongly dependent on the parameters $t_{1}, t_{3}, d_{c}$, and $\Delta$. 
At this point, we are making the distinction between the resonant modes of the two film configuration and the "resonance of the LDOS" (or, equivalently, the "resonance of the near-field spectrum"). As discussed in Sec. III A, the resonance of the two film system occurs at all frequencies along the four branches of SPhP dispersion relation. On the other hand, we refer to the resonance of the LDOS as the frequencies maximizing the near-field spectrum emitted. Such resonance of the LDOS can be estimated from SPhP dispersion relation using the following arguments. The LDOS provides a measure of the number of states, or modes, per unit volume and per unit frequency at a given spatial location, and is therefore directly proportional to $\left|d k_{\rho} / d \omega\right|$. As $k_{\rho}$ increases, the branches of SPhP dispersion relation flatten, thus implying a large $\left|d k_{\rho} / d \omega\right|$ value. We consequently expect the nearfield spectrum emitted at $\Delta$ to be maximal at the frequencies corresponding to the largest contributing parallel wavevector, $k_{\rho \text {, max }}$, where $\left|d k_{\rho} / d \omega\right|$ is the highest. By estimating the value of $k_{\rho, \max }$ as a function of $\Delta$, it is then possible to evaluate the resonance of the LDOS using SPhP dispersion relation.

The limiting frequencies of LDOS resonance can be determined by performing an asymptotic analysis of SPhP dispersion relation for a single film; these limits are also applicable for the two film configuration. We assume that only evanescent waves with penetration depth in vacuum $\delta_{2} \geq \Delta$ contribute to the LDOS calculated at $\Delta$. For a large $k_{\rho}$ value (i.e., $k_{\rho} \gg k_{v}$ ), the $z$-component of the wavevector in medium $j$, strictly defined as $k_{z j}=\sqrt{\varepsilon_{r j} k_{v}^{2}-k_{\rho}^{2}}$, can be approximated by $k_{z j} \approx i k_{\rho}$. Using the definition of penetration depth of evanescent waves, $\delta_{2} \approx\left|k_{z 2}\right|^{-1}$, we can write that the largest contributing parallel wavevector to the LDOS is $k_{\rho, \max } \approx \Delta^{-1}$. Substitution of this approximation into the dispersion relation of a single film [given below Eq. (7)] leads to

$$
\omega_{\max }^{ \pm} \approx\left[\frac{\varepsilon_{\infty} \omega_{\mathrm{LO}}^{2}+\omega_{\mathrm{TO}}^{2} \mp e^{-t_{1} / \Delta}\left(\omega_{\mathrm{TO}}^{2}-\varepsilon_{\infty} \omega_{\mathrm{LO}}^{2}\right)}{\varepsilon_{\infty}+1 \mp e^{-t_{1} / \Delta}\left(1-\varepsilon_{\infty}\right)}\right]^{1 / 2},
$$

where losses have been neglected in the dielectric function of polar crystals. Equation (8) provides an approximation of the antisymmetric and symmetric resonances of the fields above a single polar crystal film submerged in vacuum as a function of $t_{1}$ and $\Delta$. In the limiting case that $t_{1} \gg \Delta$, both modes $\omega_{\max }^{+}$ and $\omega_{\max }^{-}$maximizing the LDOS converge toward $\omega_{\text {res }}$ $\approx\left[\left(\varepsilon_{\infty} \omega_{\mathrm{LO}}^{2}+\omega_{\mathrm{TO}}^{2}\right) /\left(\varepsilon_{\infty}+1\right)\right]^{1 / 2}$, which is the resonant frequency of a single polar crystal-vacuum interface. ${ }^{7}$ The other extreme case arises when $t_{1} \ll \Delta$ leading to $\omega_{\max }^{+} \approx \omega_{\mathrm{LO}}$, and $\omega_{\max }^{-} \approx \omega_{\mathrm{TO}}$, limits that prevail regardless of the media surrounding the film. It can be shown that resonance splitting is perceptible on the LDOS profiles for a $t_{1} / \Delta$ value equal or less than unity.

The perturbation of the near-field thermal radiation spectrum emitted by film 1 due to layer 3 is analyzed hereafter as a function of three parameters: the interfilm separation gap $d_{c}$, the distance where the fields are calculated $\Delta$, and the thickness of film $3 t_{3}$. The limiting frequencies $\omega_{\mathrm{TO}}, \omega_{\mathrm{LO}}$, and $\omega_{\text {res }}$ are identified in all figures.
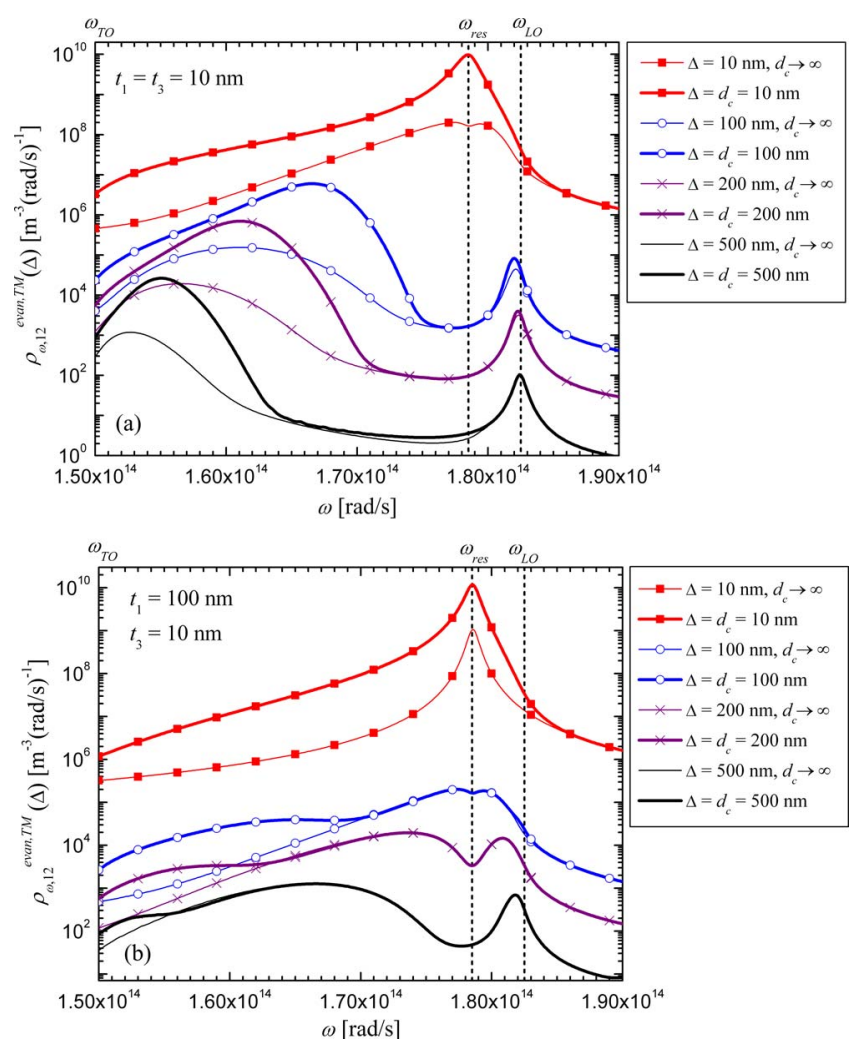

FIG. 3. (Color online) TM evanescent component of the monochromatic LDOS in the gap at $\Delta=d_{c}=10,100,200$, and $500 \mathrm{~nm}$ : (a) $t_{1}=t_{3}=10 \mathrm{~nm}$; the results are compared with a single $10 \mathrm{~nm}$ thick emitting film. (b) $t_{1}$ $=100 \mathrm{~nm}$ and $t_{3}=10 \mathrm{~nm}$; the results are compared with a single $100 \mathrm{~nm}$ thick emitting film.

\section{Impact of interfilm distance}

LDOS profiles for $d_{c}=\Delta=10,100,200$, and $500 \mathrm{~nm}$ are shown in Fig. 3(a) for $t_{1}=t_{3}=10 \mathrm{~nm}$ and Fig. 3(b) for $t_{1}$ $=100 \mathrm{~nm}$ and $t_{3}=10 \mathrm{~nm}$. In both cases, for each $\Delta$ considered, results are compared with the case $d_{c} \rightarrow \infty$ (i.e., when there is no medium 3).

When there is no film 3, the splitting of the LDOS into two distinct resonant modes is in good agreement with the approximate threshold $t_{1} / \Delta \leq 1$. As the ratio $t_{1} / \Delta$ decreases below unity, the frequencies maximizing the LDOS clearly converge toward $\omega_{\mathrm{LO}}$ and $\omega_{\mathrm{TO}}$. Physically, the $t_{1} / \Delta$ dependence on the emitted near-field spectrum can be interpreted as follows. For example, when $\Delta=10 \mathrm{~nm}$ in Fig. 3(a), using $k_{\rho, \text { max }} \approx \Delta^{-1}$, the largest contributing $K\left(K_{\max }=k_{\rho, \text { max }} / k_{v}\right)$ value to the LDOS is estimated to be 200. Inspection of the dispersion relation around this approximate limit for a single $10 \mathrm{~nm}$ thick film [Fig. 2(a)] reveals that both antisymmetric and symmetric modes have almost reached a plateau where $\left|d k_{\rho} / d \omega\right|$ is very large. As a consequence, even if the portion of dispersion relation below $K$ values of 200 contributes to the LDOS at $\Delta=10 \mathrm{~nm}$, only the region near $K_{\max }$ can be seen in Fig. 3(a) where $\left|d k_{\rho} / d \omega\right|$ is the highest. As $\Delta$ increase, the value of $K_{\max }$ decreases, the gap between $\omega^{+}$and $\omega^{-}$increases, and the near-field thermal radiation spectrum emitted clearly exhibits two distinct resonances. Also, inspection of Figs. 3(a) and 3(b) for small $t_{1} / \Delta$ ratios and $d_{c}$ $\rightarrow \infty$ shows that the LDOS resonance between $\omega_{\text {res }}$ and $\omega_{\mathrm{LO}}$ (corresponding to $\omega_{\max }^{+}$) has a higher degree of spectral co- 
herence than the LDOS resonance between $\omega_{\mathrm{TO}}$ and $\omega_{\text {res }}$ (corresponding to $\omega_{\max }^{-}$), due to greater losses of the symmetric mode. ${ }^{3}$

Figures 3(a) and 3(b) show that the presence of film 3 can enhance significantly emission by layer 1 , and perturb slightly the spectral location of the resonance of the LDOS. The enhancement of the LDOS is particularly important for $\Delta=d_{c}=10 \mathrm{~nm}$ (more than an order of magnitude), in both Figs. 3(a) and 3(b), as strong interfilm SPhP coupling increases the number of electromagnetic modes. For a $10 \mathrm{~nm}$ thick emitter and $\Delta=d_{c}=10 \mathrm{~nm}$ [Fig. 3(a)], the LDOS resonance converges to $\omega_{\text {res }}$ when medium 3 is present. Figure 2(a) shows that due to SPhP coupling, $\omega_{2}$ and $\omega_{3}$ are pushed, respectively, above $\omega^{-}$and below $\omega^{+}$, thus resulting in a maximum LDOS closer to $\omega_{\text {res }}$ than for a single film. For the $100 \mathrm{~nm}$ thick emitter, the resonance of the near-field spectrum emitted is at $\omega_{\text {res }}$ even for $d_{c} \rightarrow \infty$; the presence of medium 3 at $\Delta=d_{c}=10 \mathrm{~nm}$ spreads out the resonance over a broader spectral band (i.e., small loss of spectral coherence) as $\mathrm{SPhP}$ coupling pushes $\omega_{1}$ and $\omega_{4}$, respectively, below $\omega^{-}$ and above $\omega^{+}$of a single $10 \mathrm{~nm}$ film [see Fig. 2(b)].

As $d_{c}$ increases, SPhP coupling between the films decreases, and the enhancement of the near-field thermal radiation spectrum emitted by layer 1 consequently decreases. In Fig. 3(a) for $\Delta=d_{c}=100,200$, and $500 \mathrm{~nm}$, the presence of film 3 does not alter much the strength and spectral coherence of the LDOS resonance between $\omega_{\text {res }}$ and $\omega_{\mathrm{LO}}$ as the antisymmetric mode is not significantly altered by SPhP coupling [see Fig. 2(a) for $d_{c}=100 \mathrm{~nm}$ ]. Medium 3 mostly affects the resonance between $\omega_{\mathrm{TO}}$ and $\omega_{\text {res }}$ in terms of LDOS enhancement, spectral location, and broadening. Indeed, for a given $d_{c}$ value, $\left|d k_{\rho} / d \omega\right|$ is usually larger for $\omega_{2}$ than $\omega_{1}$. Since $\omega_{2}$ is at higher frequencies than $\omega^{-}$, the resonance between $\omega_{\text {TO }}$ and $\omega_{\text {res }}$ therefore occurs at a slightly higher frequency when film 3 is present. Similar explanations hold when the emitter is $100 \mathrm{~nm}$ thick.

The near-field thermal radiation spectrum emitted by film 1 calculated at $\Delta=d_{c}$ is always perturbed by the presence of medium 3. Indeed, if the LDOS is greater than zero at $\Delta=d_{c}$, then SPhPs emitted by film 1 necessarily couple with layer 3 thus affecting the thermal radiation field at $\Delta$.

\section{Impact of distance where the LDOS is calculated}

To analyze the influence of $\Delta$ on the LDOS profiles, we first study the case $t_{1}=t_{3}=10 \mathrm{~nm}$ and $d_{c}=100 \mathrm{~nm}$ already presented in Fig. 3(a) for $\Delta=100 \mathrm{~nm}$, and calculate the LDOS at two other locations $\Delta$ of 10 and $50 \mathrm{~nm}$. In all cases, spectral distributions of the TM evanescent component of the LDOS are compared with those without medium 3; the results are presented in Fig. 4.

Clearly, for $\Delta$ values of 10 and $50 \mathrm{~nm}$, the near-field spectrum emitted is only slightly affected by film 3 located at $d_{c}=100 \mathrm{~nm}$ above film 1 . As pointed out earlier, the perturbation of the near-field spectrum emitted by film 1 is mathematically described by the term in square brackets on the right-hand side of Eq. (5), which has a decreasing influence as $\Delta$ decreases. Physically, the $\Delta$-dependence can be explained by inspecting SPhP dispersion relation shown in Fig.

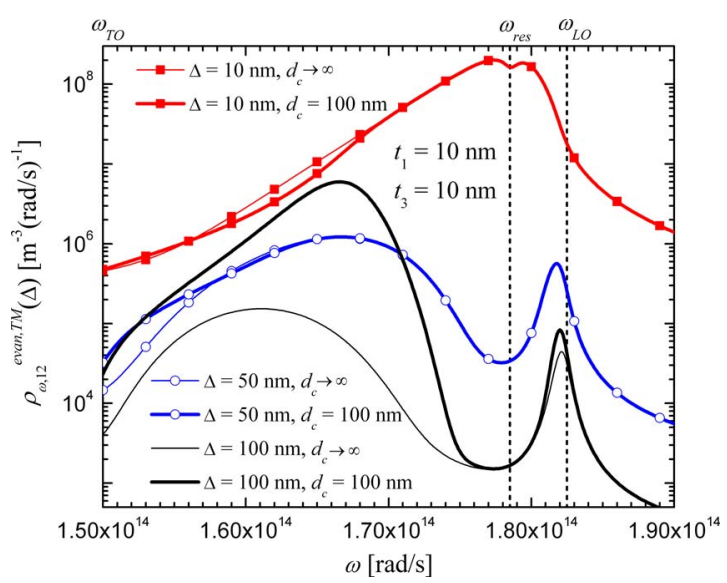

FIG. 4. (Color online) TM evanescent component of the monochromatic LDOS in the gap for $t_{1}=t_{3}=10 \mathrm{~nm}, d_{c}=100 \mathrm{~nm}$, and $\Delta=10,50$, and 100 $\mathrm{nm}$; the results are compared with a single $10 \mathrm{~nm}$ thick emitting film.

2(a). Using $k_{\rho, \max } \approx \Delta^{-1}$, the largest contributing $K$ values to the LDOS (i.e., $K_{\max }$ ) are estimated to be around 20, 40, and 200 for $\Delta$ values of 100,50 , and $10 \mathrm{~nm}$, respectively. As $K_{\max }$ increases from 20 to 40 , and from 40 to 200, the crosscoupled SPhP modes for the two layer system converge toward $\omega^{+}$and $\omega^{-}$of a single $10 \mathrm{~nm}$ thick layer. For large $K$, the branches of the dispersion relation become flattened, and $\left|d k_{\rho} / d \omega\right|$ takes very large values. Therefore, even if interfilm SPhP coupling arises for lower $K$ values, it does not have a significant influence on the near-field thermal radiation spectrum emitted by film 1 at $\Delta \ll d_{c}$ as the LDOS is dominated by $\mathrm{SPhPs}$, with small penetration depths, that do not couple with the modes of the nonemitting film.

LDOS profiles are reported in Fig. 5 for $t_{1}$ and $t_{3}$ fixed, respectively, at 100 and $10 \mathrm{~nm}, \Delta=50 \mathrm{~nm}$, and interfilm distances $d_{c}$ of 50,70,100, and $500 \mathrm{~nm}$; the results are compared with the case of a single emitting film. Moreover, the TM evanescent component of the monochromatic LDOS per unit wavevector $k_{\rho}$ is reported for the aforementioned configuration with $d_{c}=50 \mathrm{~nm}$ in Fig. 6(a), and for $d_{c}=100 \mathrm{~nm}$ in Fig. 6(b); SPhP dispersion relations are also plotted in these figures.

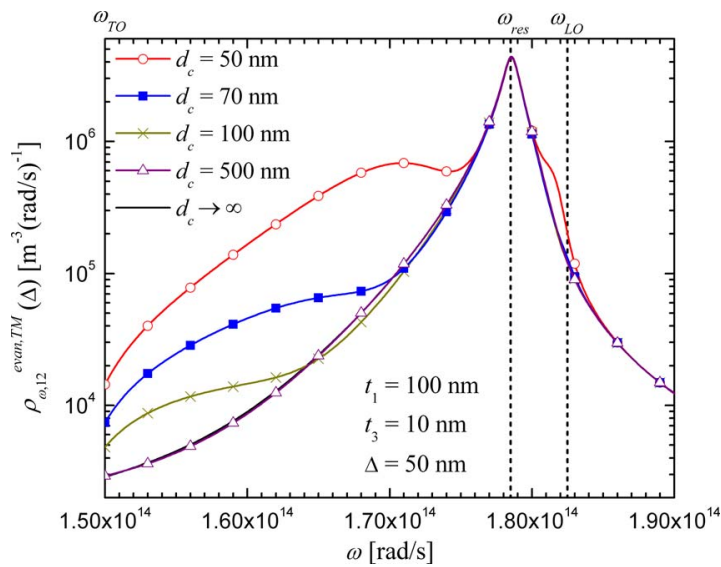

FIG. 5. (Color online) TM evanescent component of the monochromatic LDOS in the gap for $t_{1}=100 \mathrm{~nm}, t_{3}=10 \mathrm{~nm}, \Delta=50 \mathrm{~nm}$, and $d_{c}=50,70$, 100 , and $500 \mathrm{~nm}$; the results are compared with a single $100 \mathrm{~nm}$ thick emitting film. 

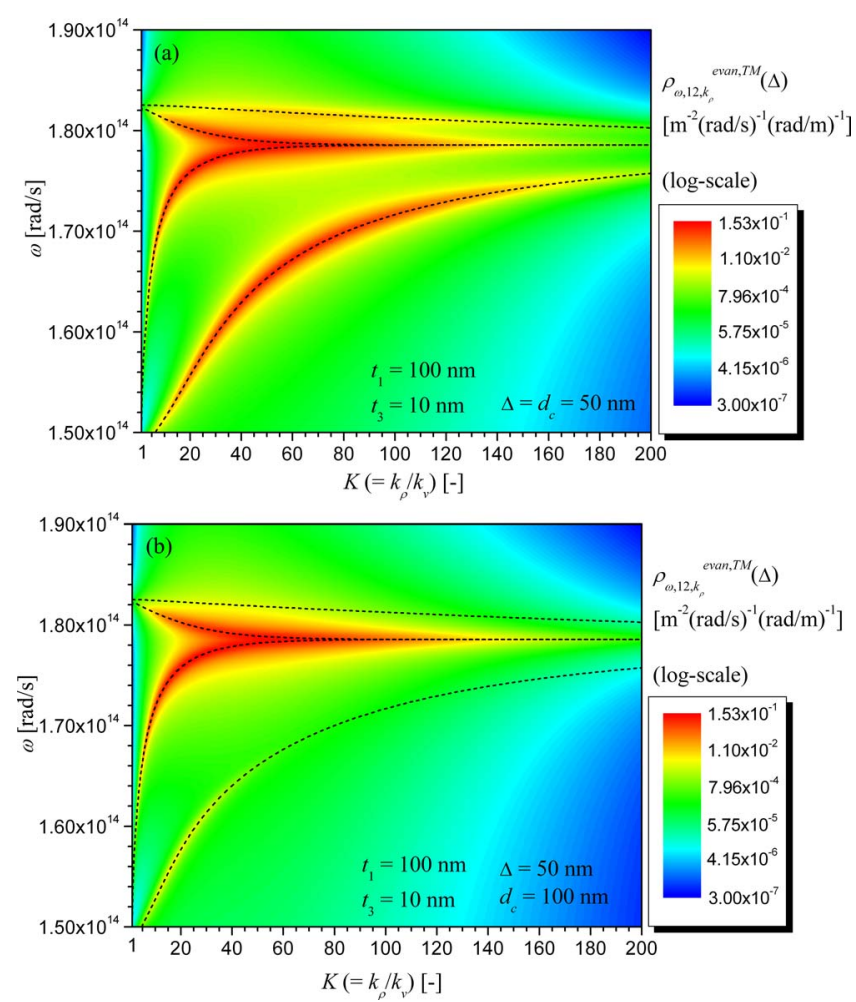

FIG. 6. (Color online) TM evanescent component of the monochromatic LDOS per unit $k_{\rho}$ (in log scale) in the gap for $t_{1}=100 \mathrm{~nm}, t_{3}=10 \mathrm{~nm}$, and $\Delta=50 \mathrm{~nm}$ : (a) $d_{c}=50 \mathrm{~nm}$. (b) $d_{c}=100 \mathrm{~nm}$.

In Fig. 5, regardless of the value of $d_{c}$, the maximum LDOS is always located at $\omega_{\text {res }}$ since for $\Delta=50 \mathrm{~nm}, K_{\max }$ is estimated around 40 where the modes $\omega_{2}$ and $\omega_{3}$ have almost reached a plateau. As $d_{c}$ decreases from 100 to $50 \mathrm{~nm}$, LDOS resonance between $\omega_{\mathrm{TO}}$ and $\omega_{\text {res }}$ shifts toward higher frequencies. Indeed, as $d_{c}$ decreases, coupling between the films becomes stronger and arises for SPhPs with lower penetration depths (i.e., larger $K$ values), such that the resonance shifts toward higher frequencies where $\left|d k_{\rho} / d \omega\right|$ is large.

Figure 6(a) for $d_{c}=\Delta=50 \mathrm{~nm}$ shows clearly that film 3 enhances near-field radiation emission around the four branches of the dispersion relation. Emission is, however, much stronger around $\omega_{\text {res }}$, where the density of electromagnetic modes is large (i.e., where $\left|d k_{\rho} / d \omega\right|$ is flat). When $d_{c}$ increases to $100 \mathrm{~nm}$ [Fig. 6(b)], the resonance at $\omega_{\text {res }}$ is still present, while the enhancement of the LDOS due to interfilm coupling has significantly decreased. It is worth noting that $K_{\max }$ estimated using $k_{\rho \text {, } \max } \approx \Delta^{-1}$ is smaller than what is shown in Figs. 6(a) and 6(b). To derive this approximation, we have used the definition of penetration depth of evanescent waves as $\delta_{j} \approx\left|k_{z j}\right|^{-1}$, which corresponds to the distance from the interface where the field amplitude has decayed to $e^{-1}$ of its value. Therefore, while $k_{\rho, \max } \approx \Delta^{-1}$ combined with $\mathrm{SPhP}$ dispersion relations can be used to explain the LDOS profiles, this procedure cannot predict with great accuracy the spectral locations of the resonant modes maximizing the near-field spectrum emitted. Such predictions, beyond the scope of this paper, would require a modification of the value of $\delta$.

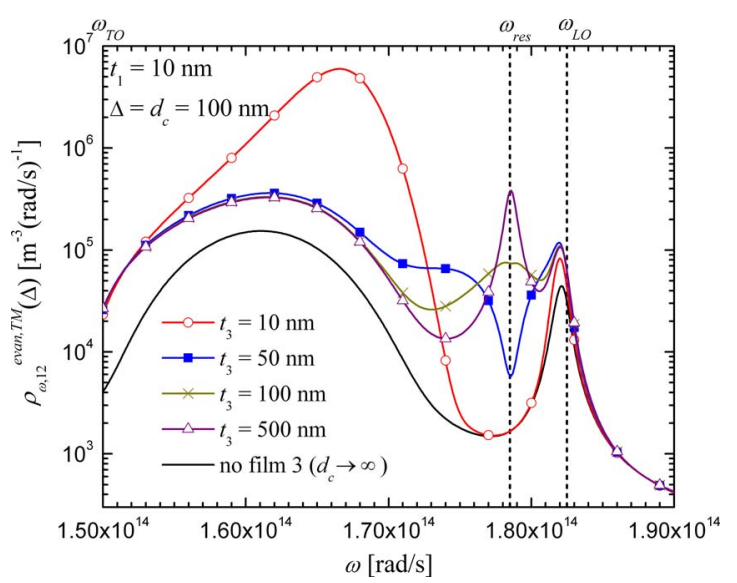

FIG. 7. (Color online) TM evanescent component of the monochromatic LDOS in the gap for $t_{1}=10 \mathrm{~nm}, \Delta=d_{c}=100 \mathrm{~nm}$, and $t_{3}=10,50,100$, and $500 \mathrm{~nm}$; the results are compared with a single $10 \mathrm{~nm}$ thick emitting film.

\section{Impact of thickness of film 3}

The TM evanescent component of the monochromatic LDOS is shown in Fig. 7 for $t_{1}=10 \mathrm{~nm}, \Delta=d_{c}=100 \mathrm{~nm}$, and varying $t_{3}$ values of $10,50,100$, and $500 \mathrm{~nm}$; the results are compared with the case when there is no film 3.

Regardless of the value of $t_{3}$, near-field thermal radiation emission is enhanced when film 3 is present compared to the case $d_{c} \rightarrow \infty$. As $t_{3}$ increases, the spectral distributions of LDOS in TM polarization vary significantly. Indeed, when the thickness of medium 3 increases, the modes $\omega_{2}$ and $\omega_{3}$ converge toward $\omega_{\text {res }}$ for lower $K$ values, while $\omega_{1}$ and $\omega_{4}$ are only slightly affected; this can be seen by comparing SPhP dispersion relations of Figs. 2(a) and 2(b) for $d_{c}=100 \mathrm{~nm}$. Therefore, it can be seen in Fig. 7 that as $t_{3}$ increases, the LDOS also increases around $\omega_{\text {res }}$. When $t_{3}=500 \mathrm{~nm}$, a resonant peak clearly emerges at $\omega_{\text {res }}$ as the modes $\omega_{2}$ and $\omega_{3}$ converge to $\omega_{\text {res }}$ for very small $K$ values, since SPhP coupling in a $500 \mathrm{~nm}$ thick medium is weak.

\section{CONCLUSIONS}

The physics of near-field thermal radiation emission by a thin layer supporting surface polaritons, in the presence of a nonemitting film also supporting surface waves, has been analyzed. This was accomplished by calculating the TM evanescent component of the monochromatic LDOS within the gap formed between two $\mathrm{SiC}$ films supporting $\mathrm{SPhPs}$ in the infrared region.

An analytical expression for the LDOS in the gap between two films has been presented for the first time. The equation has shown that due to SPhP coupling within and between the layers, the dispersion relation splits into four resonant cross-coupled modes. The analysis of the equation has also revealed that thermal emission by a film increases due to the presence of a second nonemitting layer.

The impact of interfilm separation gap $d_{c}$, the distance where the fields are calculated $\Delta$, and the thickness of the nonemitting film $t_{3}$ on the LDOS profiles have been analyzed. When $\Delta$ and $d_{c}$ were of equal lengths, the results have shown that thermal emission can significantly increase solely due to the presence of a nonemitting layer supporting SPhPs 
(more than an order of magnitude for $d_{c}=10 \mathrm{~nm}$ ). This perturbation is due to SPhP coupling between the films, which increase the number of available electromagnetic modes, while decreasing the spectral coherence of LDOS resonance. The results have revealed that the low frequency modes are mostly affected by this loss of spectral coherence. For a fixed configuration, it has been shown that as $\Delta$ decreases below $d_{c}$, the near-field spectrum is no longer affected by the presence of a second nonemitting film, since the LDOS is dominated by SPhPs with large parallel wavevectors (i.e., small penetration depths) that do not couple with the nonemitting medium. Finally, it has been shown that spectral distributions of LDOS are significantly altered as the thickness of the nonemitting film increases relative to the thickness of the emitter. This is due to the fact that as $t_{3}$ increases, a magnifying mismatch between the cross-coupled SPhP modes leading to a maximum LDOS develops, thus resulting in different spectra, which are highly dependent on the value of $t_{3}$.

It is worth noting that for thin films, spatial dispersion of the dielectric function of the materials might be important, and consequently nonlocal effects will be investigated in a future research effort. Moreover, when applying the fluctuational electrodynamics theory, the media are assumed to be in local thermodynamic equilibrium. For films with thicknesses of the order of few nanometers, this assumption might be questionable. On the other hand, this theory, built on macroscopic electrodynamics, is currently the only tool available to treat near-field thermal radiation emission. Validation of the application of fluctuational electrodynamics to very thin layers should come from experiments.

The work presented here provides guidelines on how near-field thermal radiation emission by a film is affected by the presence of another body. Starting from this physical analysis, we aim to develop an engineering approach to tune near-field thermal radiation emission via multilayered media, without necessarily going through complex inversion procedures, which will be applied to design optimal nanoscale-gap TPV power generators.

\section{ACKNOWLEDGMENTS}

This work is partially sponsored by the U.S. Department of Energy Grant No. DE-FG02-07ER46375 and the Ken- tucky Science and Engineering Foundation Grant No. KSEF1718-RDE-011. Partial support for M.P.M. is received from the FP-7-PEOPLE-IRG-2008 Grant No. 239382 at Ozyegin University.

${ }^{1}$ S.-A. Biehs, D. Reddig, and M. Holthaus, Eur. Phys. J. B 55, 237 (2007).

${ }^{2}$ S.-A. Biehs, Eur. Phys. J. B 58, 423 (2007).

${ }^{3}$ K. Joulain and C. Henkel, Appl. Phys. B: Lasers Opt. 93, 151 (2008).

${ }^{4}$ J. Drevillon, Ph.D. thesis, Université de Nantes, 2007.

${ }^{5}$ P. Ben-Abdallah, K. Joulain, J. Drevillon, and G. Domingues, Appl. Phys. Lett. 94, 153117 (2009).

${ }^{6}$ C. J. Fu and W. C. Tan, J. Quant. Spectrosc. Radiat. Transf. 110, 1027 (2009).

${ }^{7}$ M. Francoeur, M. P. Mengüç, and R. Vaillon, Appl. Phys. Lett. 93, 043109 (2008).

${ }^{8}$ P. Ben-Abdallah, K. Joulain, J. Drevillon, and G. Domingues, J. Appl. Phys. 106, 044306 (2009).

${ }^{9}$ K. L. Kliewer and R. Fuchs, Phys. Rev. 144, 495 (1966).

${ }^{10}$ K. L. Kliewer and R. Fuchs, Phys. Rev. 150, 573 (1966).

${ }^{11}$ K. L. Kliewer and R. Fuchs, Phys. Rev. 153, 498 (1967).

${ }^{12}$ E. N. Economou, Phys. Rev. 182, 539 (1969).

${ }^{13}$ D. Sarid, Phys. Rev. Lett. 47, 1927 (1981).

${ }^{14}$ J. J. Burke, G. I. Stegeman, and T. Tamir, Phys. Rev. B 33, 5186 (1986).

${ }^{15}$ J. Dionne, L. A. Sweatlock, H. A. Atwater, and A. Polman, Phys. Rev. B 72, 075405 (2005).

${ }^{16}$ S. A. Maier, Plasmonics: Fundamentals and Applications (Springer, New York, 2007).

${ }^{17}$ M. Laroche, R. Carminati, and J.-J. Greffet, J. Appl. Phys. 100, 063704 (2006).

${ }^{18}$ K. Park, S. Basu, W. P. King, and Z. M. Zhang, J. Quant. Spectrosc. Radiat. Transf. 109, 305 (2008).

${ }^{19}$ S. M. Rytov, Y. A. Kravtsov, and V. I. Tatarski, Principles of Statistical Radiophysics (Springer-Verlag, Berlin, 1987), Vol. 3.

${ }^{20}$ M. Francoeur and M. P. Mengüç, J. Quant. Spectrosc. Radiat. Transf. 109, 280 (2008)

${ }^{21}$ M. Francoeur, M. P. Mengüç, and R. Vaillon, J. Quant. Spectrosc. Radiat. Transf. 110, 2002 (2009).

${ }^{22}$ J. E. Sipe, J. Opt. Soc. Am. B 4, 481 (1987).

${ }^{23} \mathrm{P}$. Yeh, Optical Waves in Layered Media (Wiley, Hoboken, NJ, 2005).

${ }^{24} \mathrm{~K}$. Joulain, Topics in Applied Physics: Microscale and Nanoscale Heat Transfer (Springer, Berlin, 2007), Vol. 107, p. 107.

${ }^{25}$ K. Joulain, J.-P. Mulet, F. Marquier, R. Carminati, and J.-J. Greffet, Surf. Sci. Rep. 57, 59 (2005).

${ }^{26}$ E. D. Palik, Handbook of Optical Constants of Solids (Academic, San Diego, CA, 1998), Vol. 1.

${ }^{27}$ M. A. Gilmore and B. L. Johnson, J. Appl. Phys. 93, 4497 (2003).

${ }^{28}$ J. Chen, G. A. Smolyakov, S. R. J. Brueck, and K. J. Malloy, Opt. Express 16, 14902 (2008). 